\title{
Facile Method for Obtaining Gold-Coated Polyester Surfaces with Antimicrobial Properties
}

\author{
M. Drobota $\mathbb{D}^{1},{ }^{1}$ M. Butnaru $\mathbb{D}^{1,2}$ N. Vornicu $\mathbb{D}^{3},{ }^{3}$ O. Plopa $\mathbb{D}^{4},{ }^{4}$ and M. Aflori $\mathbb{D}^{1}$ \\ 1 "Petru Poni" Institute of Macromolecular Chemistry, 41A, Grigore Ghica Voda Alley, Iasi 700487, Romania \\ ${ }^{2}$ Department of Biomedical Sciences "Grigore T. Popa" University of Medicine and Pharmacy, 9-13, Kogalniceanu Street, \\ Iasi 700115, Romania \\ ${ }^{3}$ Metropolitan Center of Research T.A.B.O.R, The Metropolitanate of Moldavia and Bukovina, Closca 9 Street, Iasi 700066, Romania \\ ${ }^{4}$ SC Intelectro Iasi SRL, Iancu Bacalu no. 3 Street, Iasi 700029, Romania \\ Correspondence should be addressed to M. Aflori; maflori@icmpp.ro
}

Received 14 December 2019; Revised 13 February 2020; Accepted 14 March 2020; Published 2 April 2020

Guest Editor: Anindya Basu

Copyright (C) 2020 M. Drobota et al. This is an open access article distributed under the Creative Commons Attribution License, which permits unrestricted use, distribution, and reproduction in any medium, provided the original work is properly cited.

\begin{abstract}
The antimicrobial and antifungal activity of polymers used in medical devices has been extensively studied due to the growing impact of hospital-related infections in patients. The ideal biocidal polymeric materials should be very effective in the microorganism's inhibition, not toxic to the human body, and environmentally friendly. In this context, this work is aimed at obtaining antimicrobial and antifungal properties at the polyester film surfaces without introducing toxic effects. Poly (ethylene terephthalate) (PET) films were functionalized with Ar plasma and then immersed in a solution containing gold nanoparticles (AuNps). The results demonstrated the appearance of the hydrophilic groups on the film surface after modification of PET film by plasma Ar treatment and the formation of the polar groups such as $\mathrm{C}=\mathrm{O}, \mathrm{COO}-$, and $\mathrm{OH}$, which then reacted with AuNps. The changes induced in the treated polymer samples were investigated in terms of AuNp adsorption efficiency on polyester film by contact angle, profilometry, Scanning Electron Microscopy (SEM), Attenuated Total Reflectance Spectroscopy-Fourier Transform Infrared (ATR-FTIR), and X-ray Photoelectron Spectroscopy (XPS) measurements. The morphological and structural analyses have shown a good adhesion of AuNps at treated film surfaces. The results of biocompatibility antimicrobial and antifungal tests proved the nontoxic behavior of the sample and its good antimicrobial and antifungal activity.
\end{abstract}

\section{Introduction}

The functional groups on a polymer film surface are very important in tailoring the properties for targeted biomedical applications $[1,2]$. The surface characteristics depend on the balance of hydrophobic-hydrophilic of the polymeric material, which induces properties such as adsorption, adhesion, permeability, wettability, and other important parameters for various applications [3-6]. The surface activation is a common way to promote polymers with properties for target applications. Various methods of surface functionalization, such as plasma activation, UV activation, laser activation, electron-beam irradiation, and chemical activation, improved the polar group concentration at the polymer's surface, enriching their biocompatibility [7-9]. Polyethylene terephthalate (PET) is one of the most studied polymers for flexible substrates used in biosensing platforms [10-12]. PET is often used to layer functional components such as metal coatings, metal oxides, conducting polymers, and nanoparticles to form thin structures that can easily conform to biological surfaces $[13,14]$. From our knowledge, there are not many studies that address the subject of PET enriched with gold nanoparticles [15], although the surface properties prove to be radically improved by these treatments. A flexible molecular imprinted electrochemical biosensor using $\mathrm{Au} / \mathrm{PPy}$ nanowires was proposed for dopamine sensing [16], but Au nanowires were first grown on the flexible Au-coated PET substrates. Other authors [17] described the fabrication of a simple and inexpensive glucose sensor based on PET film with a gold electrode. The presence of gold nanoparticles in biosensors can improve its sensitivity due to the generation of a biocompatible 
microenvironment, greatly increasing the amount of immobilized biomolecules on the electrode surface [18, 19].

The introduction of antimicrobial properties into medical devices has been extensively studied to control the growing problem of hospital-related infections. A greater number of studies have successfully exploited polymeric and inorganic nanoparticles (NPs) [20-23]. Among those, gold nanoparticles (AuNPs) are one of the most investigated tools in nanomedicine. These particles have been used as therapeutic agents [24], diagnosis agents [25], and imaging agents [26, 27]. Due to their nanoscale size with dimensions comparable to those of the biological compounds, AuNps present remarkable physicochemical properties (which are different from those of the corresponding bulk materials). The AuNps act as a multimodal tool in enhancing scaffold properties, cell differentiation, and intracellular growth factor. Because the materials containing AuNps present antimicrobial activity, they are very appealing for the applications requiring biocidal surfaces [28].

The present study is aimed at developing a sensitive biocidal surface using plasma activation of PET film surfaces. Under Ar plasma treatment, the surface polar group concentration increases [29]. It is known that the increase of the polymer surface energy charges is due to the incorporation of the $\mathrm{C}-\mathrm{O}, \mathrm{C}=\mathrm{O}$, and $\mathrm{O}-\mathrm{C}=\mathrm{O}$ groups after functionalization [30-32]. The treatment time cannot be too long but must be enough for optimal surface activation. That means that the surface must be only activated, not degraded, to keep the surface properties unaltered [33]. After activation, the films were introduced in $\mathrm{Au}$ buffer solution. The activated PET film surface with $\mathrm{Au}$ particles was investigated by contact angle measurement (WCA), Attenuated Total Reflectance Spectroscopy-Fourier Transform Infrared (ATR-FTIR), Scanning Electron Microscopy Imaging (SEM), and X-ray Photoelectron Spectroscopy (XPS). Cell growth assays on these films have been performed using fibroblast cells to prove the efficiency of our studies in biological applications.

\section{Experimental}

2.1. Materials. Poly (ethylene terephthalate) (PET) film biaxial oriented with $30 \mu \mathrm{m}$ thickness (Tg 80) was obtained from TEROM, Iasi, Romania. AuNps of $15 \mathrm{~nm}$ diameter, present in stabilized suspension in $0.1 \mathrm{mM}$ PBS Au reactant free, was purchased from Sigma-Aldrich. The fibroblast cells used in this work were isolated by explant method, from Albino rabbit skin dermis, according to the animal welfare requirements and ethical approval of the Ethical Committee of the Grigore T. Popa University of Medicine and Pharmacy of Iasi. Briefly, $1 \mathrm{~cm}^{2}$ of the rabbit skin was sterilized using a 3-step washing procedure in the Dulbecco's Modified Eagle Media (DMEM-Sigma) with decreasing antibiotic concentration as following: 1st step-10 min shaking in $4 \%$ penicillin/streptomycin/neomycin (PSN) solution (Sigma), 2nd step-10 min shaking in 2\% PSN solution, and 3th step-10 min shaking in DMEM without PSN. After the last washing step, the hypodermic part of the skin was removed, while the dermis and the epidermis were cut in small pieces, 1-2 $\mathrm{mm}$ size. The skin pieces were plated with the dermis part down on a thin layer of the bovine fetal serum (BFS-Sigma), spread on the culture surface of the $25 \mathrm{~cm}^{2}$ TC-treated cell culture flask, and then covered with a small amount of DMEM supplied with $15 \%$ BFS and 1\% PSN. The seeded explants were incubated for approximately two weeks at the $37^{\circ} \mathrm{C}, 97-98 \%$ atmospheric humidity, and $5 \% \mathrm{CO}_{2}$, until the confluent cell monolayer was formed around each tissue explant. The primary dermal cells from the explants were cultured for several generations through standard cell techniques [34]. For the biocompatibility experiments, the 4 and 5 cell passages were used.

For antimicrobial activity tests of the samples, four fungi (Aspergillus niger ATCC 53346, Fusarium ATCC 20327, Penicillium chrysogenum ATCC 20044, and Alternaria alternata ATCC 8741) from pure culture and two bacteria (Pseudomonas aeroginosa ATCC 27813 and Bacillus sp. ATCC 31073) species were chosen. [35].

Microbial suspensions were prepared in accordance with the direct colony method; the microbial suspension consists of a mixture of 6 microorganisms. The successive dilution procedure has been used to prepare the suspension of microorganisms [36]. The final load of as prepared stock inoculum was $1 \times 10^{4}$ cells $/ \mathrm{ml}$. The number of viable microorganisms is achieved by increasing dilutions in a liquid medium, in which they are in contact with fixed quantities from the microbial culture. For these determinations, we used Petri dishes with culture medium agar-Sabouraud and agar-agar and simple Broth, at $37^{\circ} \mathrm{C}$ from Merck. The inhibition zone measurement was performed using the Olympus SZX 160 microscope with QuickPHOTO micro 2.3 processing software.

2.2. Surface Treatments. The steps of polymer surface treatment are described in Figure 1. The PET films were cleaned, washed with ethanol, dried at room temperature, cut into $5 \times 5 \mathrm{~cm}$ pieces, and then introduced in a radiofrequency plasma reactor (Emitech K1050 Plasma Asher, Emitech Ltd, UK). After the ignition of argon plasma (gas purity $99.997 \%$, power $30 \mathrm{~W}$, and the pressure of 10-2 mbar), from a large series, two treatment times were chosen because of their low degradability rate $[37,38]$ and high performance of introducing active groups at polymer surface at 3 and $5 \mathrm{~min}$. (Figure 1(a)).

The PET films with activated surface were immersed for $12 \mathrm{~h}$ into the colloid solution of $\mathrm{Au}$ nanoparticles (Figure 1(b)). The studied samples were PET (pristine PET), PET-3Au (PET treated for $3 \mathrm{~min}$ in the plasma reactor and then immersed in $\mathrm{Au}$ solution), and PET-5Au (PET treated for $5 \mathrm{~min}$ in the plasma reactor and then immersed in $\mathrm{Au}$ solution). To avoid the excess of nonbound Au nanoparticles at the treated surfaces, all samples were cleaned with MilliQ water.

2.3. Characterization Methods. In the present work, the surface morphology of the samples was investigated by using contact angle, SEM Scanning Electron Microscope analysis, and profilometry, while the structural changes were recorded by using FTIR and XPS methods. 


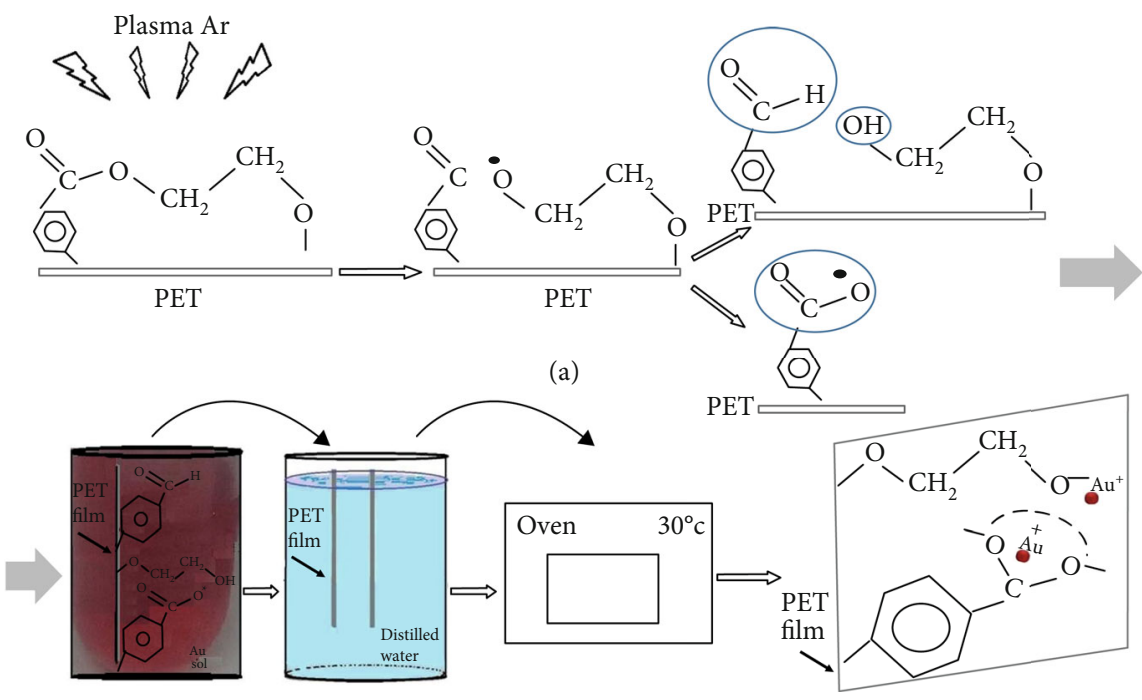

(b)

FIGURE 1: Schematic treatment mechanism.

Static contact angle values were evaluated by the sessile-drop method, with a CAM-101 (KSV Instruments Ltd., Helsinki, Finland). The system equipped with a liquid dispenser for contact angle measurements includes a video camera and analysis software for drop shape (KSV CAM Optical Contact Angle and Pendant Drop Surface Tension Software, version 3.99, KSV Instruments Ltd., Helsinki, Finland). The liquids used as solvents for these studies are double-distilled water and ethylene glycol. For each kind of liquid, three different regions of the surface for each liquid were selected at room temperature.

Scanning Electron Microscope (SEM) micrographs were recorded with a Quanta 200 scanning probe microscope (FEI Company, Brno, Czech Republic).

The infrared spectra were investigated using a LUMOS FT-IR Microscope (Bruker Optik GmbH, Ettlingen, Germany) with an Attenuated Total Reflectance (ATR) reflection module containing a diamond crystal and single reflection at $45^{\circ}$ angle and software for spectral processing OPUS 8. The sample surface was scanned in the 600$4000 \mathrm{~cm}^{-1}$ range. All spectra were collected by cumulating 64 scans at a resolution of $2 \mathrm{~cm}^{-1}$. The spectra were recorded at a constant temperature of $25^{\circ} \mathrm{C}$.

The X-ray photoelectron spectra (XPS) were recorded on a KRATOS Axis Nova (Kratos Analytical, Manchester, United Kingdom) instrument equipped with a monochromatized $\mathrm{Al} \mathrm{K} \alpha \mathrm{X}$-ray source $(1486 \mathrm{eV})$. The wide scan was recorded by using a monochromatic X-ray beam focused on a $0.8 \times 0.2 \mathrm{~mm}$ area of the surface, in various locations on each sample. The XPS survey spectra were collected in the range of -10 to $1200 \mathrm{eV}$, with a resolution of $1 \mathrm{eV}$. For each sample, five measurements were performed.

$\mathrm{Au}$ colloid solutions before and after polymer treatment were analyzed by Ultraviolet-visible (UV-vis) spectrophotometer (Barloworld Scientific Ltd, Dunmow, Essex, UK).

Film roughness was determined using a Tencor AlphaStep D-500 stylus profiler (KLA Tencor Corporation, Milpitas, CA, USA) at $1000 \mu \mathrm{m}$ scan length and $100 \mu \mathrm{m} / \mathrm{s}$ scan speed. The arithmetic average of the absolute values of the profile heights over the evaluation length $\mathrm{Ra}$ was measured by applying a stylus force of $2.3 \mathrm{mg}$ and a long-range cutoff filter of $25 \mu \mathrm{m}$.

Cell-grow images were recorded using an inVia ${ }^{\mathrm{TM}}$ confocal Raman microscope spectrometer (Renishaw plc, Gloucestershire, United Kingdom) equipped with a Leica DM2700 microscope with $5 \mathrm{x}, 20 \mathrm{x}$, and 100x objectives.

2.4. Antimicrobial and Biocompatibility Tests. Antimicrobial and antifungal activity was tested by determining the minimum inhibitory concentration (MIC) using the disc diffusion method. All MIC range was followed according to the NCCLS guidelines (NCCLS, 1997). In the Petri plates, the standard culture medium was seeded with the microorganism suspension throughout the surface. The diffusion disk is represented by samples 3 and 5 with dimensions of $1 \mathrm{~cm}^{2}$. The MIC evaluation was made in relation to the blank sample. The inoculated plates were incubated at $32^{\circ} \mathrm{C}$ for 7 days. The first observations were made after 24 hours by measuring the inhibition zone and the final measuring after 7 days.

2.4.1. Cell culture. The sterilized and equilibrated polymer pieces with a diameter of $10 \mathrm{~mm}$ were placed on the bottom of the wells of a 48-well culture plate. The fibroblast suspension in DMEM supplemented with $10 \%$ BFS and 1\% PSN, at the concentration of $2 \times 104$ cells/material piece, was added over polymer and cultured at $37^{\circ} \mathrm{C}$ temperature and $5 \%$ $\mathrm{CO}_{2}$ atmosphere for 24 hours. After 24 hours of cell seeding, the materials with the attached cells were moved to a new culture plate and cultured in the same condition until at list day 2. The attached cells on the material surface were fixed using $4 \%$ paraformaldehyde solution and analyzed by confocal Raman microscope spectrometer (Renishaw plc, Gloucestershire, United Kingdom) equipped with a Leica DM2700 microscope with $5 x, 20 x$, and 100x objective microscopy. The cytotoxicity of each material sample was determined by 
TABLE 1: Surface roughness and wettability results.

\begin{tabular}{|c|c|c|c|c|c|c|c|c|}
\hline \multirow{2}{*}{ Sample } & \multirow{2}{*}{$\begin{array}{l}\text { Roughness } \\
\mathrm{Ra}(\mathrm{nm})\end{array}$} & \multicolumn{7}{|c|}{ Contact angle measurements parameters } \\
\hline & & $\theta_{\mathrm{w}}$ & $\theta_{\mathrm{EG}}$ & W & $\gamma_{\mathrm{SV}}^{\mathrm{p}}$ & $\gamma_{\mathrm{SV}}{ }^{\mathrm{d}}$ & $\gamma_{\mathrm{SV}}$ & $\gamma_{\mathrm{SL}}$ \\
\hline PET & 22.95 & 79.67 & 54.75 & 114.82 & 95.57 & 7.06 & 102.63 & 10.99 \\
\hline $\mathrm{PET}-3 \mathrm{Au}$ & 33.73 & 51.49 & 27.20 & 137.55 & 108.42 & 1.43 & 109.85 & 22.76 \\
\hline PET-5Au & 57.74 & 54.35 & 22.46 & 140.07 & 121.77 & 3.52 & 125.29 & 22.95 \\
\hline
\end{tabular}

the viability of the cells growing with the materials. For cell viability measuring, the widely used MTT assay was chosen [39]. Briefly, the sterilized and equilibrated polymer pieces with a diameter of $5 \mathrm{~mm}$ were incubated with fibroblast suspension at the cell concentration of $1 \times 10^{4}$ cells/material piece. After 48 hours of cell seeding, the MTT reaction was performed. The experimental results have been expressed as a percent of control cell viability (cells growing without any material).

\section{Results and Discussion}

3.1. Surface Wettability and Morphology. The water contact angle of the pristine PET film of about $80^{\circ}$ indicated the hydrophobic character of the sample. The reactive groups on the PET oxidized surface were due to plasma gases, allowing the introduction of the Au particles onto the pretreated polymer substrate. If the surface has hydrophobic character, the water will not be able to penetrate the hollows and pores on the rough surface and will keep a resting state in a semisolid and semiair plane surface, which will increase the contact angle significantly [40]. The surface has a big improvement in wettability after the plasma treatment, the surface character being modified from hydrophobic to a hydrophilic one and an increase in the surface roughness being noticed. The differences in the contact angle values show that the presence of the polar groups like hydroxyl and carboxyl groups after surface activation improves the wettability of the surface film.

The reason for these observed behaviours is the amount of bonded broken fragments of the macromolecules and surfaces the irregularities restricting reorientation of the groups which contain the oxygen from the surface of the film. These are the results of the plasma interaction with the polymer surface.

The values of the water contact angle for different treatment times decreased with increasing treatment time, remaining in the hydrophilic area. From the OwensWendt-Rabel $[41,42]$, the values of the static contact angle $\left(\theta_{\mathrm{w}}\right.$ for water and $\theta_{\mathrm{EG}}$ for ethylene glycol) were used to estimate the wettability and surface tension of the solid surface. Based on these measurements, some parameters such as surface-free energy $\left(\gamma_{\mathrm{SV}}\right)$, solid-liquid interfacial tension $\left(\gamma_{\mathrm{S}}\right.$ ), or work of adhesion $(W)$ were determined. The increase of the plasma treatment time causes an increase of the surface roughness parameters $\mathrm{Ra}$ (Table 1 ), measured with the profiler. In contrast, the water will penetrate and fill up most of the hollows, and the Au nanoparticles which have an intrinsically hydrophilic material will form a surface with one solid part and other liquid parts and therefore leading to a low water contact angle compared to the pristine sample.

The values listed in Table 1 revealed that both plasma treatments significantly increased the surface energy, mainly due to the increase in its polar component, the highest value being obtained for PET-5Au sample. The active sites created by the plasma treatment on the polymer surface ensured appropriate interactions and sufficient bonding sites which allows for anchoring of the gold nanoparticles (reflected in $\theta_{\mathrm{w}}$ ). The amount of AuNps at the polymer surface is increasing with the increasing of the plasma treatment time. This evolution correlates with the evolution of the interfacial tension $\left(\gamma_{\mathrm{SL}}\right)$, which increases with the increase of the Au density on the surface (nanoparticles having very high surface energy) [43].

According to this method, depending on the number of functional groups on the surface of the polymer, when the values of one of the components decrease, the other one will increase. Thus, for studied PET samples, the disperse component has lower values than the polar one, so there are more hydrophilic groups than hydrophobic on the surface. The interfacial energy value may be high or low depending on the attraction forces between the fluid molecules and the solid surface. Thus, the greater the force of attraction between the liquid molecules and the polymer surface is, the higher the amount of energy will be recorded, and the drop of liquid will adhere less to a treated polymer surface.

The plasma modifications enrich the surface with polar groups, such as different types of carbonyl, hydroxyl, and hydroperoxide groups [37]. After treatments, free surface energy $\gamma_{\mathrm{SV}}$ and the interfacial energy $\gamma_{\mathrm{SL}}$ increase compared with the pristine film, and Au particles can be easily immobilized on the surface of materials due to their interaction with polar groups.

From SEM images (see Figure 2), both times of treatment evidence a dense distribution [44] of the gold nanoparticles on the surface for all samples.

The two-step treatment leads to a significant change in surface morphology, resulting in the increased adhesion properties of the treated surface samples compared to the pristine one, with the formation of Au-polyester bound. The plasma treatment followed by immersion in a solution containing Au nanoparticles created nanoscale irregularities on the material surface. In most cases, the Au nanoparticles can be associated with the presence of a cluster, which influences the electrostatic interaction characteristics of the sample by electronic transfer $[45,46]$.

The initial solution with Au nanoparticles has a bright red colour and presents peak absorption at $520 \mathrm{~nm}$ in the UV spectrum (Figure 3). After the immersion of plasma- 


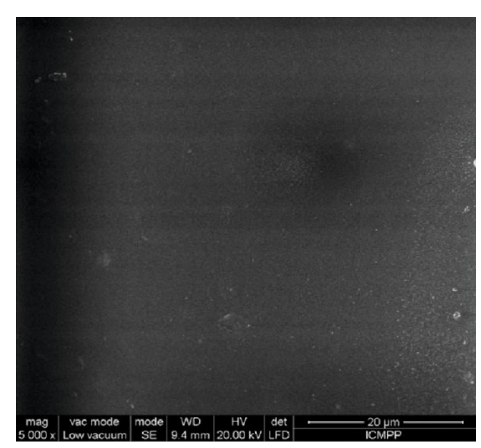

(a)

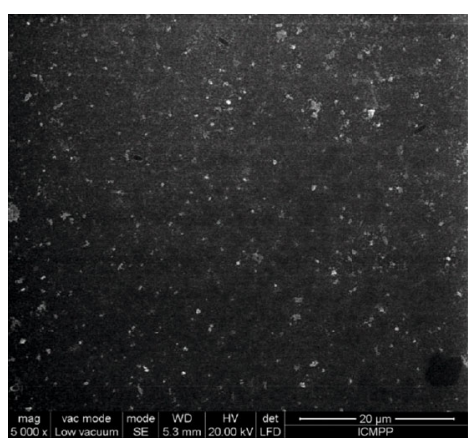

(b)

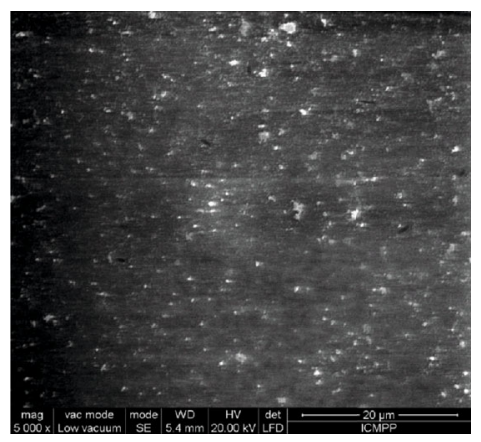

(c)

FIGURE 2: SEM of images of PET (a) untreated and anchoring the Au particles after plasma action for (b) $3 \mathrm{~min}$ and (c) $5 \mathrm{~min}$.

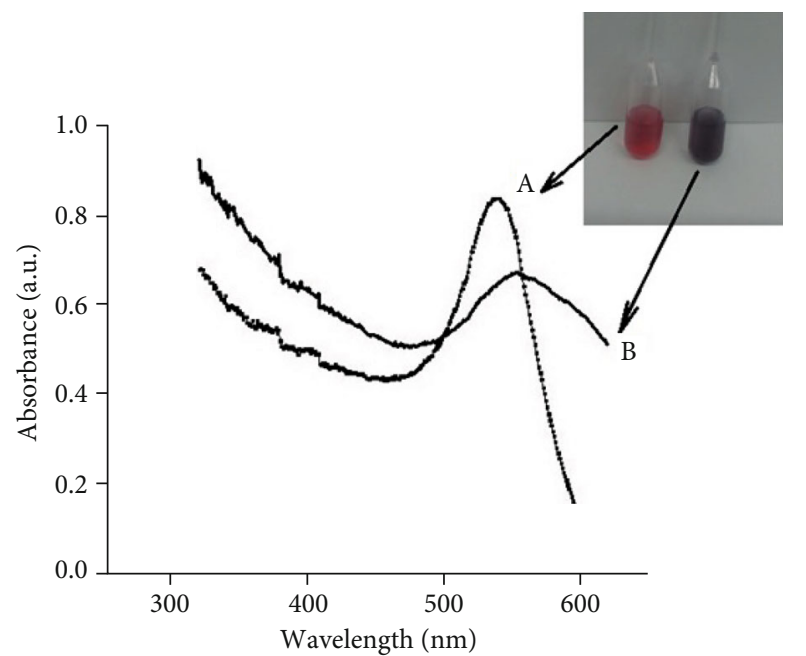

FIGURE 3: UV-vis spectra of the gold nanoparticle solution: (A) before and (B) after the immersion of plasma-treated PET films.

treated PET films, the solution presents a violet colour usually associated with the agglomeration of nanoparticles, and the peak absorption shifts to $534 \mathrm{~nm}$ [47].

3.2. Surface Chemistry. The ATR-FTIR spectra of the pristine PET (Figure 4) contain characteristic PET vibrations at $1717 \mathrm{~cm}^{-1}(\nu \mathrm{C}=\mathrm{O}$ this vibration is the overlapping of peaks of the ester groups), $1102 \mathrm{~cm}^{-1}$ (C-O stretching), the stretching bands at $1409 \mathrm{~cm}^{-1}$ (aromatic skeletal with $\mathrm{C}=\mathrm{C}$ groups from internal band), the bending vibration at 1340 and $1370 \mathrm{~cm}^{-1} \mathrm{w}\left(\mathrm{CH}_{2}\right), \quad 1018 \mathrm{~cm}^{-1} \delta(\mathrm{C}-\mathrm{H}), \quad 873 \mathrm{~cm}^{-1}$, and $729 \mathrm{~cm}^{-1}$ for $\gamma(\mathrm{C}-\mathrm{H})$ and $\gamma(\mathrm{C}-\mathrm{O})$. The PET film treated in an inert gas-like Ar induces polar components.

After functionalization, the strong bands of polar groups at $3340 \mathrm{~cm}^{-1}$ from $\mathrm{O}-\mathrm{H}$ stretching and bond of the ester groups of $\mathrm{C}=\mathrm{O}-\mathrm{O}$ at $1238 \mathrm{~cm}^{-1}$ appeared in the FTIR spectrum. At $1648 \mathrm{~cm}^{-1}$, the characteristic vibration from polar groups indicates the presence of the $\mathrm{Au}$ nanoparticles attached on the film surface and in the interaction with carbonyl groups. The metallic $\left(\mathrm{Au}^{\circ}\right)$ gold is involved in those reactions, forming a certain complex. The negative groups on the PET surface were detected by ATR-FTIR spectroscopy, and in Figure 4, the spectra show a new peak at
$1620 \mathrm{~cm}^{-1}$ attributed to COO- band after interactions with $\mathrm{Au}$ [48]. ATR-FTIR spectroscopy confirms the interaction between carbonyl group with Au particles and the apparition of a complex (see Figure 5).

The interactions of the $\mathrm{Au}^{+}$ions with carboxylate groups (R-COO- $\mathrm{Au}^{+}$interactions) also have as result the appearance of a new complex. In the case of interaction with $\mathrm{Au}$, the binding energy of the oxygen depends on the parity of the number of electrons in the cluster: when the number of electrons is odd, the interaction is stronger, due to a smaller ionization potential.

The vibration signals in the spectra of the treated samples, located at 1527 and $1585 \mathrm{~cm}^{-1}$, show the formed carboxylate anions. In the ATR-FTIR, spectra are presented the vibrations from $\mathrm{C}-\mathrm{H}$ aliphatic stretching $\left(3000-2850 \mathrm{~cm}^{-1}\right)$, and $\mathrm{C}-\mathrm{H}$ bend $\left(1470-1370 \mathrm{~cm}^{-1}\right)$ indicating the attachment of the particles and ions. A new absorption appears at $2150 \mathrm{~cm}^{-1}$. These absorptions were attributed to CO absorptions, which were in interaction with Au. These considerations have been taken as some observations from researchers $\sim 2100 \mathrm{~cm}^{-1}$ have been reported in the literature in FTIR spectra from CO-free molecules in the presence of $[\mathrm{PVP}] /[\mathrm{Au}]$ ratios of 100 [49].

3.2.1. XPS Analysis. The changes in PET surface composition after treatments were revealed by XPS measurements, which demonstrate the presence of C1s, O1s, and Au4f peaks [20] in the recorded spectra. The results of XPS analysis are in concordance with the data from ATR-FTIR: the Au concentration increased with increasing treatment time. From the XPS data, the amount of Au presented on the treated polyester surface is higher in the case of PET-5Au treatment compared to PET-3Au. The XPS spectra indicate that the concentration of atomic percent of $\mathrm{C}$ and $\mathrm{O}$ decreases, in parallel with increasing the concentration of $\mathrm{Au}$ particles. The elemental composition and the ratio of elements are summarized in Table 2 . The presence of the new oxygen-containing groups on the PET film surfaces is reflected in the change of the $\mathrm{O} / \mathrm{C}$ atomic ratio, as well as in the improvement of the surface hydrophilicity. This atomic ratio explains the hydrophilic growth from 0.34 to 0.35 [50].

Figure 6 shows the XPS spectra of all the studied samples. The plasma treatment induces the presence of polar groups 


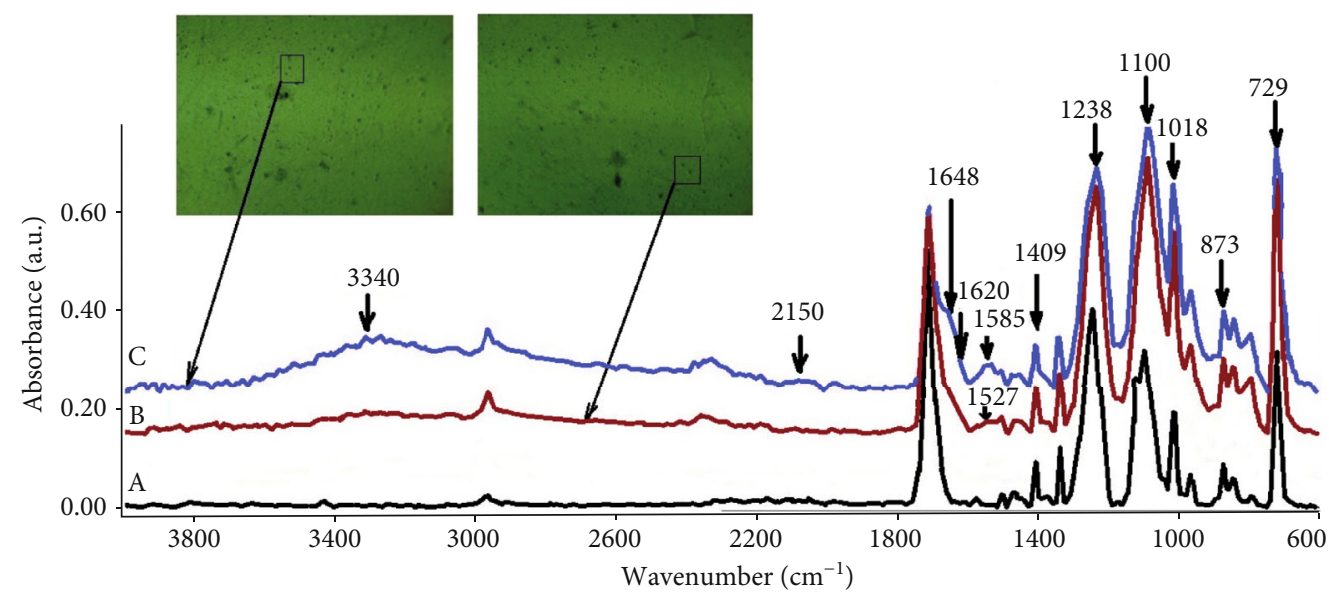

Figure 4: ATR-FTIR spectra (A) PET film, (B) PET-3Au, and (C) PET-5Au.

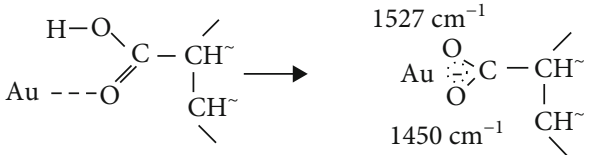

Figure 5: Possible interaction between $\mathrm{Au}$ particles and polar groups after activated surface PET film.

on the surface film (surface enriched with O- and COO-), and the $\mathrm{Au}$ atoms act as electron acceptors. An electron transfers from oxygen to gold particles because on the surface we found species with oxygen such as carboxy, peroxy, and hydroxyl, a charge transfer from the substrate to the Au particles could explain this behavior. XPS experiments demonstrated that the particles were partially oxidized.

The Au4f7/2_5/2 core-level peak was found into two doublets assigned to the $\mathrm{Au}^{0}$ located at $82.9 \mathrm{eV}$ and the $\mathrm{Au}^{3+}$ at higher binding energy side $(86.6 \mathrm{eV})$. We assume that on the surface of the polymer, we will find metallic $\mathrm{Au}, \mathrm{Au}^{+}$, and $\mathrm{Au}^{3+}$. These values from the XPS analysis data revealed a shift of $4 \mathrm{f}$ to about $82 \mathrm{eV}$, which can be attributed to metallic $\mathrm{Au}$. These values of the XPS values indicate a state of $\mathrm{Au}$ metal even if it is in a state of oxidation, so a shift of value to smaller values was observed in the specialized literature by other researchers [51].

Thus, it is noticeable that upon oxidation of the Au metal, it moves to lower values of the connecting energy (eV) [52]. This behavior was observed at the interaction of Au particles that interact with a negatively charged present on the surface become active, especially when interacting with oxygencontaining species.

The polar groups play an important role in anchoring the Au particles on PET. The appearance of complexes at polymer surface after the interaction with the colloidal solution containing $\mathrm{Au}$ nanoparticles was also demonstrated by UV measurements (Figure 2) and was accompanied by a change in the gold solution color (from red to indigo).

The species $\mathrm{Au}^{3+}$ adsorbed on PET samplefunctionalized surfaces led to the change in the solution
TABLE 2: Element concentrations of $\mathrm{C}, \mathrm{O}$, and $\mathrm{Au}$ from XPS measurements.

\begin{tabular}{lccccc}
\hline \multirow{2}{*}{ Sample } & \multicolumn{5}{c}{ Element concentration (at.\%) } \\
& $\mathrm{C}$ & $\mathrm{O}$ & $\mathrm{Au}$ & $\mathrm{O} / \mathrm{C}$ & $\mathrm{Au} / \mathrm{C}$ \\
\hline PET & 74.76 & 25.24 & - & 0.34 & - \\
PET-3 Au & 74.45 & 26.41 & 1.14 & 0.36 & 0.02 \\
PET-5 Au & 71.92 & 25.09 & 2.99 & 0.35 & 0.04 \\
\hline
\end{tabular}

color. This fact can be correlated with the particle size. When the surface of the polymer has been activated, the increase in activity per unit surface area has been achieved by the charge on the surface with more electronic charge activity that may induce an increase in the concentration of nanoparticles attached on the membrane and in the same time, more crowded areas may appear.

The gold coordination state is influenced by the support that is loaded with different oxygen-containing species in interaction with $\mathrm{Au}$ and present onto the polymer surface due to the plasma treatment. The peak of the Au metallic from $4 \mathrm{f}$ is shifted due to the electron transfer from Au. Some authors [47] concluded that $\mathrm{Au}^{\delta(+)}$ was converted to $\mathrm{Au}^{\delta(-)}$ by electron transfer induced by the different functional species containing oxygen. The oxidation state is influenced by the distance between species and the spatial orientation of the Au particles.

Several aspects could be highlighted by FTIR and XPS measurements: first of all, a successful immobilization of the stable gold nanoparticles at the polyester surface at different concentrations, depending on the plasma treatment time (which induced certain active group in certain concentration), can be observed. On the other hand, an interaction mechanism can be proposed based on the interaction of gold with $\mathrm{O}$ - and $\mathrm{COO}-$ groups from the plasma-treated polymer surface by electronic transfer. After plasma treatment, $\mathrm{OH}$ groups are present at the polymer surface, from the atmosphere and can interact with gold forming nonstable species such as $\mathrm{HO}-\mathrm{Au}-\mathrm{CO}$, which will act as intermediary species. 


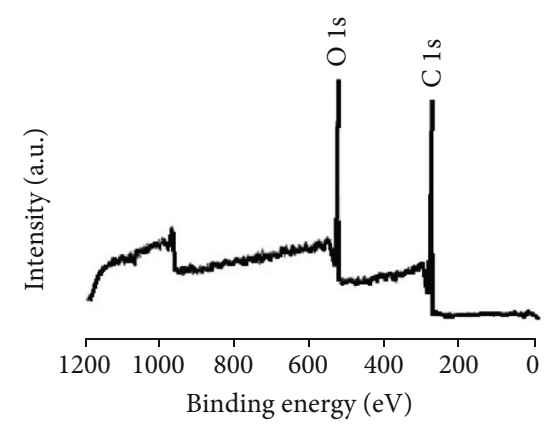

(a)

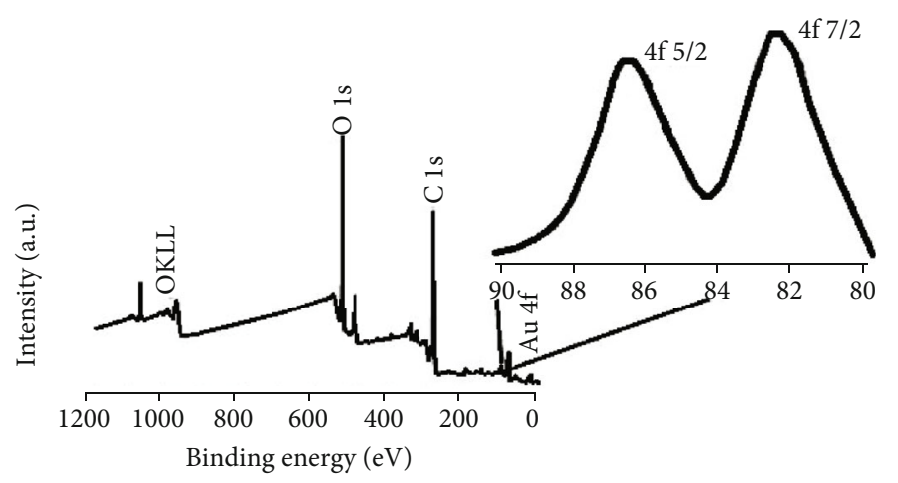

(b)

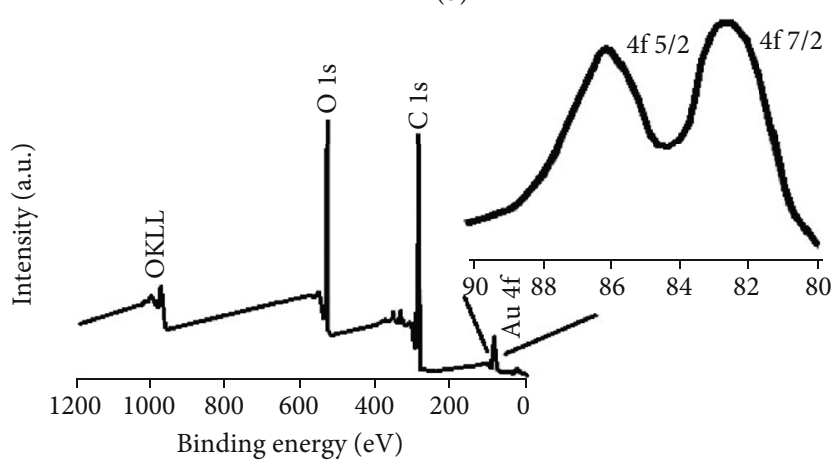

(c)

Figure 6: XPS spectra of (a) PET pristine, (b) PET-3Au, and (c) PET-5Au.

3.3. Cell Population Tests. The distribution and the cell population are developing in a manner depending on surface topology and roughness. The balance between hydrophilicity and hydrophobicity due to the rearrangement of the polar groups is depending on the surface treatment conditions. The surface roughness influences the surface wettability, i.e., by improving the geometric conformation, better exposure of the active sites is induced, and allowing better adhesion of the cells.

Cellular adhesion to materials is initially mediated by the first layer previously adsorbed onto the surface of the material. The formation of the contacts between cells and support occurs when the binding of fibroblast cell is realized in different points, determining the interaction between the cells and the polymer support.

Figure 7 presents confocal microscope images of the biocompatibility test results on studied samples populated with fibroblast. The cell's activity after $48 \mathrm{~h}$ was compared to one of the unmodified samples (PET), revealing an increased cell proliferation. The cell viability at $48 \mathrm{~h}$ of fibroblast incubation was of $100 \%$ for the untreated PET, $81.9 \%$ for $3 \mathrm{~min}$, and $85.07 \%$ for $5 \mathrm{~min}$ of plasma treatment, followed by Au particle immobilization. The surface chemistry, the morphology, the surface energy, and the hydrophilicity play an important role in cell adhesion, thus, influencing the cell binding on the substrate and the ability to proliferate [53]. The surface energy of PET-studied samples plays an important role in cell adhesion: more fibroblasts can adhere and spread widely on the more hydrophilic polymer surface [54].

3.3.1. Results of the Antimicrobial Activities. The screening results indicate that the two analysed samples, for the antimicrobial activities, MIC values for the tested polyester film samples are PET-3Au and PET-5Au, have good antibacterial and antifungal activity and sample PET-5Au has a $2 \mathrm{~mm}$ inhibition area and for the PET-3Au sample a $0.5 \mathrm{~mm}$. The evaluation of the tests was performed to compare with the PET sample, the antimicrobial activity of PET-3Au and 


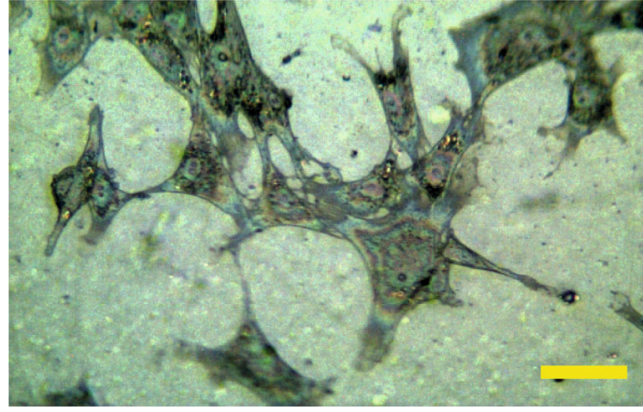

(a)

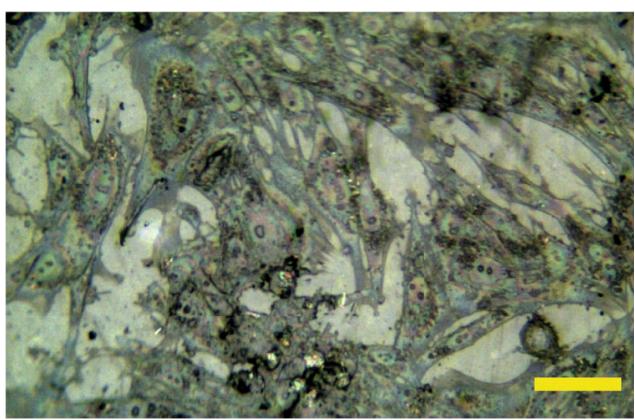

(b)

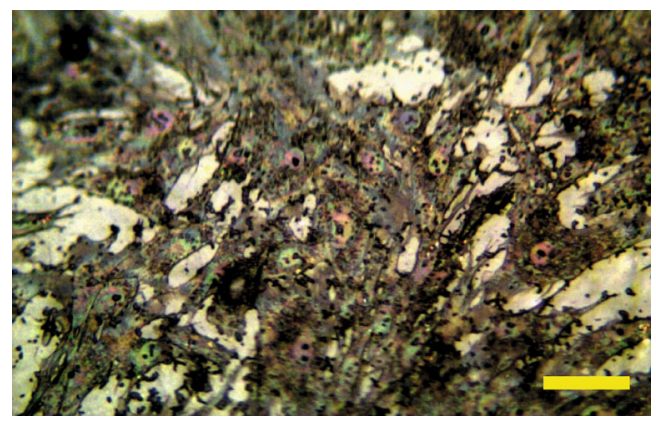

(c)

Figure 7: Images of fibroblast cells grown for $48 \mathrm{~h}$ on (a) PET untreated film, (b) PET-3Au, and (c) PET-5Au (scale $100 \mu \mathrm{m}$ ).

TABle 3: Results of "in vitro" antimicrobial activity testing of compounds and inhibition zones (mm) after 24 hours.

\begin{tabular}{|c|c|c|c|c|c|c|}
\hline \multirow{2}{*}{ Sample } & \multicolumn{3}{|c|}{ Fungi } & \multicolumn{3}{|c|}{ Bacteria } \\
\hline & Aspergillus niger & Fusarium & Penicillium chrysogenum & Alternaria alternata & Bacillus sp. & Pseudomonas aeruginosa \\
\hline PET (martor) & - & - & - & - & - & - \\
\hline PET-3Au & 0.5 & 0.5 & 0.5 & 0.5 & 0.5 & 0.5 \\
\hline PET-5Au & 2 & 2 & 2 & 2 & 2 & 2 \\
\hline
\end{tabular}

No inhibition zone.

PET-5Au samples was significantly higher, the bacteria being killed under the same experimental conditions, proving that Au loading on the surface is an effective strategy. The results confirm that the resistance to the bacteria may be based on the interaction of the components, groups present on the surface, the ions AuO-, AuNps, and O- groups which increase the ability to form the hydrogen bonds and finally to determinate the results of the antimicrobial (Table 3) and antifungal activities which are present in Figure 8. Thus, the biological activity can be explained based on the structure of the films modified with Au nanoparticles: the gold is immobilized by physical bounds only at the surface of the polymer in a thin layer (not in whole material), due to the presence of the functionalities after plasma treatment. In this particular case, we expect to have migration of gold into the medium. It is known that the nanoparticles when nonagglomerated are toxic to gram-negative bacteria. Bactericidal effects were due inducing from the NPs and the antimicrobial activity, which means that the influences that affect the bacterial and fungi integrity are the major causes of bacterial death. Indeed, there is kept a remarkable capacity to determinate the NP-generated oxidative stress, induced from certain groups containing metal migrate from the surfaces of the polymeric films.

The attachment and the extent mechanisms of the cells on the polymer surface are directed by a combination of physical and chemical factors that arise from the adsorption process of nanoparticles. Besides, cell-surface attachment is controlled by surface characteristics like charge chemistry, the degree of hydrophobicity, roughness, the specific geometry (macro, micro, and nano), and the free energy. The plasma treatment leads to the appearance of oxygen-rich polar groups which caused the reorientations on the surface. Compared with the untreated PET, the treated samples showed an increase of the hydrophilicity and roughness of the surface, for both PET-3Au and PET-5Au. In general, an increase in biocompatibility can be attributed to the increase of surface polarity and to the presence of oxygen-rich groups, as well as to an increase in its roughness [55]. On the other hand, long treatment duration indicates a surface contraproductive for cell adhesion and proliferation. An excessive number of new functional groups introduced to the surface 


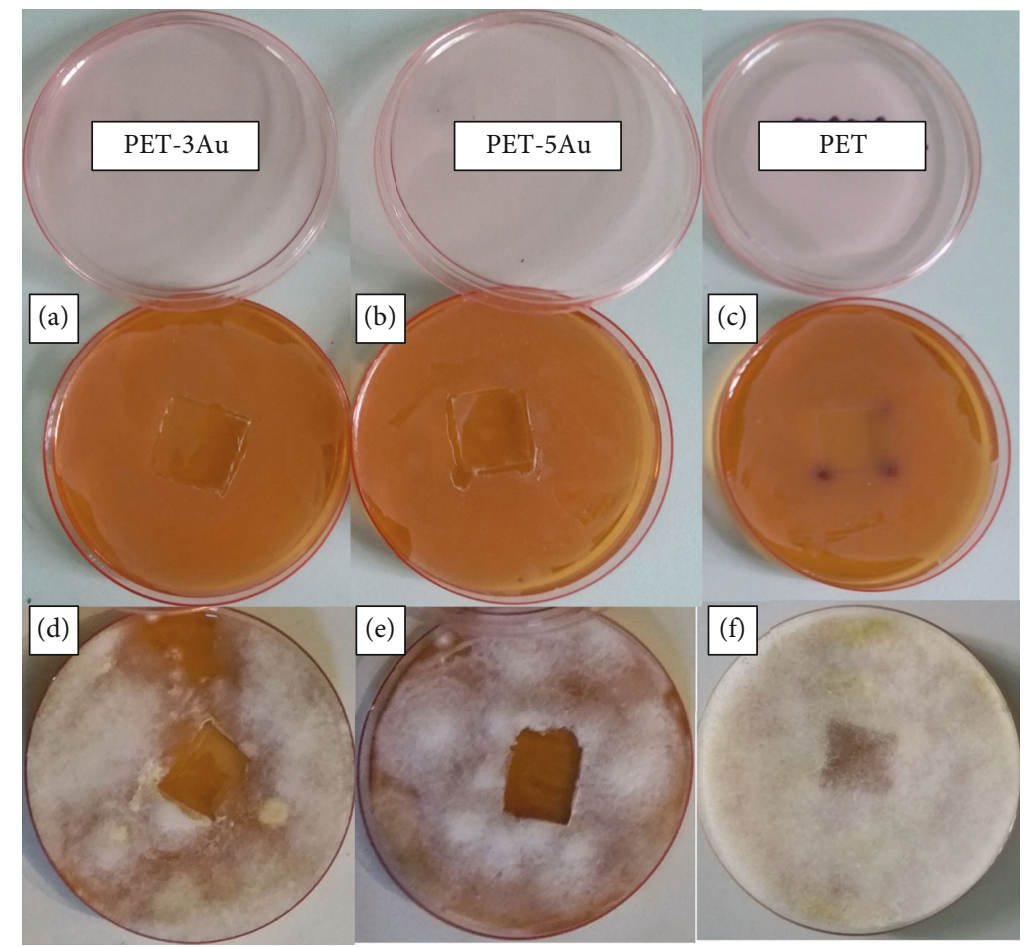

FIgure 8: Antimicrobial activity of AuNPs for 1 day (a, b, c) and 7 days (d, e, f).

induces a decrease in the number of adhered cells $[56,57]$ as oxidative stress. It is known that the fact that a strongly hydrophilic surface, for example, does not adsorb proteins from the blood, while a strongly hydrophobic one preferentially binds albumin, due to its high concentration in blood [58]. According to other studies, the number of cells appears to be invariant regardless of the material and the roughness value, and that cell adhesion mechanisms are not influenced by the roughness of biomaterial surface, below a critical value of about $11-13 \mu \mathrm{m}$ [59]. This surface roughness could be in this case a negligible effect on the fibroblast adhesion process at the surface [60]. The effect of surface roughness on cell adhesion has been evaluated by other researchers [61-63]. The amount of nanoparticles bound to the modified surface depends not only on the plasma treatment parameters but also on the type of the grafted nanoparticles. Metal nanoparticles (Ag, Au, Pt, and Pd) were synthesized by PVD technique into glycerol, but from the cytotoxicity point of view AuNPs may be considered as most cell-friendly [63]. For the particle with $16 \mathrm{~nm}$ size (similar size as in our case), these researchers found a similar trend for the cell adhesion and spreading. In our case, the biocompatibility tests indicated a value of $100 \%$ for cell population on pristine PET, $81.9 \%$ for PET-3Au, and $85.07 \%$ for PET-5Au. The cell adhesion in our case is dependent on the critical concentration value at the polymer surface and on the size of nanoparticles. For the sample PET-3Au, the small amount of adhered cells can be explained by the combined effect of surface oxidative stress and roughness. For PET-5Au sample, it seems that oxidative stress and cytotoxicity do not influence cell adhesion as much as it does influence the amount of nanoparticles, showing an increase in the cell population.

\section{Conclusions}

The main goal of this paper was to perform a study concerning the efficiency of the plasma treatment in anchoring $\mathrm{Au}$ particles on polymeric support in terms of obtaining antimicrobial and antifungal surface properties. Different chemical and morphological characterization revealed the changes in surface properties which were correlated with further antimicrobial and antifungal properties. The surface energy of studied samples plays an important role in cell adhesion: more fibroblasts can adhere and spread widely on the more hydrophilic polymer surface.

The method proved to be facile and nontoxic for epithelial cells. The screening results indicate that the two analyzed samples, PET-3Au and PET-5Au, have good antibacterial and antifungal activity, and sample PET-5Au has a $2 \mathrm{~mm}$ inhibition area and for PET-3Au sample of $0.5 \mathrm{~mm}$. The results confirm the resistance to the bacteria may be based on the interaction of the groups present on the surface (the ions AuO-, AuNps, and O- groups) which increases the ability to form the hydrogen bonds and finally to determinate the results of the antimicrobial activities. Thus, the biological activity can be explained by taking in consideration the structure of the films modified with $\mathrm{Au}$ nanoparticles. Those properties are useful for many biomedical applications, including biosensors in direct contact with the skin.

\section{Data Availability}

The data used to support the findings of this study are available from the corresponding author upon request. 


\section{Conflicts of Interest}

The authors declare no conflicts of interest.

\section{Acknowledgments}

The authors acknowledge the financial support of this research through the Project "Partnerships for knowledge transfer in the field of polymer materials used in biomedical engineering" ID P_40_443, Contract no. 86/8.09.2016 and SMIS 105689, cofinanced by the European Regional Development Fund and by the Competitiveness Operational Programme 2014-2020, Axis 1, Research, Technological Development and Innovation, in support of economic competitiveness and business development, Action 1.2.3 Knowledge Transfer Partnerships.

\section{References}

[1] S. Haridoss and M. M. Perlman, "Chemical modification of near-surface charge trapping in polymers," Journal of Applied Physics, vol. 55, no. 5, pp. 1332-1338, 1984.

[2] K. S. Iyer and I. Luzinov, "Effect of macromolecular anchoring layer thickness and molecular weight on polymer grafting," Macromolecules, vol. 37, no. 25, pp. 9538-9545, 2004.

[3] K. Cho, K. Takenaka, Y. Setsuhara, M. Shiratani, M. Sekine, and M. Hori, "Effects of irradiation with ions and photons in ultraviolet-vacuum ultraviolet regions on nano-surface properties of polymers exposed to plasmas," Japanese Journal of Applied Physics, vol. 51, no. 1S, article 01AJ02, 2012.

[4] M. Donegan, V. Milosavljevic, and D. P. Dowling, "Activation of PET using an RF atmospheric plasma system," Plasma Chemistry and Plasma Processing, vol. 33, no. 5, pp. 941-957, 2013.

[5] T. Homola, J. Matoušek, B. Hergelová, M. Kormunda, L. Y. L. Wu, and M. Černák, "Activation of poly(ethylene terephthalate) surfaces by atmospheric pressure plasma," Polymer Degradation and Stability, vol. 97, no. 11, pp. 2249-2254, 2012.

[6] D. Kontziampasis, V. Constantoudis, and E. Gogolides, "Plasma directed organization of nanodots on polymers: effects of polymer type and etching time on morphology and order," Plasma Processes and Polymers, vol. 9, no. 9, pp. 866872, 2012.

[7] I. Junkar, A. Vesel, U. Cvelbar, M. Mozetič, and S. Strnad, "Influence of oxygen and nitrogen plasma treatment on polyethylene terephthalate (PET) polymers," Vacuum, vol. 84, no. 1, pp. 83-85, 2009.

[8] S. Große-Kreul, C. Corbella, A. von Keudell, B. Ozkaya, and G. Grundmeier, "Surface modification of polypropylene (PP) by argon ions and UV photons," Plasma Processes and Polymers, vol. 10, no. 12, pp. 1110-1119, 2013.

[9] A. E. Kolosov, V. I. Sivetskii, E. P. Kolosova et al., "Creation of structural polymer composite materials for functional application using physicochemical modification," Advances in Polymer Technology, vol. 2019, Article ID 3501456, 12 pages, 2019.

[10] P. H. Lau, K. Takei, C. Wang et al., "Fully printed, high performance carbon nanotube thin-film transistors on flexible substrates," Nano Letters, vol. 13, no. 8, pp. 3864-3869, 2013.

[11] C. Liao, M. Zhang, M. Y. Yao, T. Hua, L. Li, and F. Yan, "Flexible organic electronics in biology: materials and devices," Advanced Materials, vol. 27, no. 46, pp. 7493-7527, 2015.
[12] M. Segev-Bar and H. Haick, "Flexible sensors based on nanoparticles," ACS Nano, vol. 7, no. 10, pp. 8366-8378, 2013.

[13] F. F. Vidor, T. Meyers, and U. Hilleringmann, "Flexible electronics: integration processes for organic and inorganic semiconductor-based thin-film transistors," Electronics, vol. 4, no. 3, pp. 480-506, 2015.

[14] Z. W. Zhao, X. J. Chen, B. K. Tay, J. S. Chen, Z. J. Han, and K. A. Khor, "A novel amperometric biosensor based on $\mathrm{ZnO}$ :Co nanoclusters for biosensing glucose," Biosensors and Bioelectronics, vol. 23, no. 1, pp. 135-139, 2007.

[15] I. V. Korolkov, D. B. Borgekov, A. A. Mashentseva et al., "The effect of oxidation pretreatment of polymer template on the formation and catalytic activity of Au/PET membrane composites," Chemical Papers, vol. 71, no. 12, pp. 2353-2358, 2017.

[16] W. R. Huang, Y. L. Chen, C. Y. Lee, and H. T. Chiu, "Fabrication of gold/polypyrrole core/shell nanowires on a flexible substrate for molecular imprinted electrochemical sensors," RSC Advances, vol. 4, no. 107, pp. 62393-62398, 2014.

[17] Y. Wang, X. Wang, W. Lu, Q. Yuan, Y. Zheng, and B. Yao, “A thin film polyethylene terephthalate (PET) electrochemical sensor for detection of glucose in sweat," Talanta, vol. 198, pp. 86-92, 2019.

[18] K. Z. Liang, J. S. Qi, W. J. Mu, and Z. G. Chen, "Biomolecules/gold nanowires-doped sol-gel film for label-free electrochemical immunoassay of testosterone," Journal of Biochemical and Biophysical Methods, vol. 70, no. 6, pp. 1156-1162, 2008.

[19] X. He, R. Yuan, Y. Chai, and Y. Shi, "A sensitive amperometric immunosensor for carcinoembryonic antigen detection with porous nanogold film and nano-Au/chitosan composite as immobilization matrix," Journal of Biochemical and Biophysical Methods, vol. 70, no. 6, pp. 823-829, 2008.

[20] M. Drobota, L. M. Gradinaru, C. Ciobanu, and I. Stoica, "Collagen immobilization on poly(ethylene terephthalate) and polyurethane films after UV functionalization," Journal of Adhesion Science and Technology, vol. 29, no. 20, pp. 22082219, 2015.

[21] M. Drobota, M. Aflori, L. M. Gradinaru et al., "Collagen immobilization on ultraviolet light-treated poly(ethylene terephthalate)," High Performance Polymers, vol. 27, no. 5, pp. 646-654, 2015.

[22] N. de Geyter, R. Morent, C. Leys, L. Gengembre, and E. Payen, "Treatment of polymer films with a dielectric barrier discharge in air, helium and argon at medium pressure," Surface and Coating Technology, vol. 201, no. 16-17, pp. 7066-7075, 2007.

[23] M. Aflori, M. Drobota, D. G. Dimitriu, I. Stoica, B. Simionescu, and V. Harabagiu, "Collagen immobilization on polyethylene terephthalate surface after helium plasma treatment," Materials Science and Engineering B, vol. 178, no. 19, pp. 13031310, 2013.

[24] L. K. Xie, Q. L. Dai, G. B. du, Q. P. Deng, and G. L. Liu, "Study on surface modification of polyethylene Terephthalate(PET) film by RF-Ar $/ \mathrm{O}_{2}$ plasma treatment," Applied Mechanics and Materials, vol. 200, pp. 194-198, 2012.

[25] N. Inagaki, S. Tasaka, K. Narushima, and H. Kobayashi, "Surface modification of PET films by pulsed argon plasma," Journal of Applied Polymer Science, vol. 85, no. 14, pp. 2845-2852, 2002.

[26] A. Tautzenberger, Kovtun, and Ignatius, "Nanoparticles and their potential for application in bone," International Journal of Nanomedicine, vol. 7, pp. 4545-4557, 2012. 
[27] R. Sensenig, Y. Sapir, C. MacDonald, S. Cohen, and B. Polyak, "Magnetic nanoparticle-based approaches to locally target therapy and enhance tissue regeneration in vivo," Nanomedicine, vol. 7, no. 9, pp. 1425-1442, 2012.

[28] S. Fleischer and T. Dvir, "Tissue engineering on the nanoscale: lessons from the heart," Current Opinion in Biotechnology, vol. 24, no. 4, pp. 664-671, 2013.

[29] V. E. Santo, M. T. Rodrigues, and M. E. Gomes, "Contributions and future perspectives on the use of magnetic nanoparticles as diagnostic and therapeutic tools in the field of regenerative medicine," Expert Review of Molecular Diagnostics, vol. 13, no. 6, pp. 553-566, 2013.

[30] T. M. Sun, Y. C. Wang, F. Wang et al., "Cancer stem cell therapy using doxorubicin conjugated to gold nanoparticles via hydrazone bonds," Biomaterials, vol. 35, no. 2, pp. 836-845, 2014.

[31] T. L. Halo, K. M. McMahon, N. L. Angeloni et al., "Nanoflares for the detection, isolation, and culture of live tumor cells from human blood," Proceedings of the National Academy of Sciences of the United States of America, vol. 111, no. 48, pp. 17104-17109, 2014.

[32] A. de la Zerda, S. Prabhulkar, V. L. Perez et al., "Optical coherence contrast imaging using gold nanorods in living mice eyes," Clinical and Experimental Ophthalmology, vol. 43, no. 4, pp. 358-366, 2015.

[33] K. Hayashi, M. Nakamura, and K. Ishimura, "Near-infrared fluorescent silica-coated gold nanoparticle clusters for X-ray computed tomography/optical dual modal imaging of the lymphatic system," Advanced Healthcare Materials, vol. 2, no. 5, pp. 756-763, 2013.

[34] R. I. Freshney, Culture of animal cells. A manual of basic technique and specialized applications, Wiley \& Sons, Inc., Hoboken, New Jersey, USA, 6th edition, 2010.

[35] J. E. Bennett, "Aspergillosis," in Harrison's Principles of Internal Medicine, K. J. Isselbacher, R. D. Adams, E. Braunwald, R. G. Petersdorff, and J. D. Wilson, Eds., pp. 742-744, McGraw-Hill, New York, 1980.

[36] M. Nucci and E. Anaissie, "Fusarium infections in immunocompromised patients," Clinical Microbiology Reviews, vol. 20, no. 4, pp. 695-704, 2007.

[37] D. P. Vasilkin, T. G. Shikova, V. A. Titov, S. A. Smirnov, and L. A. Kuzmicheva, "Influence the loading effect on modification of PET film and fiber by argon Plasma," Journal of Physics Conference Series, vol. 789, article 012068, 2017.

[38] V. Kotál, V. Švorčík, P. Slepička et al., "Gold coating of poly(ethylene terephthalate) modified by argon plasma," Plasma Processes and Polymers, vol. 4, no. 1, pp. 69-76, 2007.

[39] M. Iacob, A. Bele, X. Patras et al., "Preparation of electromechanically active silicone composites and some evaluations of their suitability for biomedical applications," Materials Science and Engineering: C, vol. 43, pp. 392-402, 2014.

[40] R. E. Johnson and R. H. Dettre, "Wetting of low energy surfaces," in Wettability, J. C. Berg, Ed., pp. 1-74, Dekker, New York, NY, 1993.

[41] B. Bhushan and Y. C. Jung, "Natural and biomimetic artificial surfaces for superhydrophobicity, self- cleaning, low adhesion, and drag reduction," Progress in Materials Science, vol. 56, no. 1, pp. 1-108, 2011.

[42] L. Makkonen, "A thermodynamic model of contact angle hysteresis," Journal of Chemical Physics, vol. 147, no. 6, article 064703, 2017.
[43] N. S. Kasálková, P. Slepička, Z. Kolská et al., "Cell adhesion and proliferation on polyethylene grafted with au nanoparticles," Nuclear Instruments and Methods in Physics Research Section B, vol. 272, pp. 391-395, 2012.

[44] V. Švorčík, N. Kasálková, P. Slepička et al., "Cytocompatibility of $\mathrm{Ar}^{+}$plasma treated and $\mathrm{Au}$ nanoparticle-grafted PE," Nuclear Instruments and Methods in Physics Research Section B, vol. 267, no. 11, pp. 1904-1910, 2009.

[45] D. Knittel and E. Schollmeyer, "Functional group analysis on oxidized surfaces of synthetic textile polymers," Talanta, vol. 76, no. 5, pp. 1136-1140, 2008.

[46] G. Zhang, M. du, Q. Li et al., "Green synthesis of Au-Ag alloy nanoparticles using Cacumen platycladi extract," RSC Advances, vol. 3, no. 6, pp. 1878-1884, 2013.

[47] G. Yang, W. S. Chang, and D. T. Hallinan Jr., "A convenient phase transfer protocol to functionalize gold nanoparticles with short alkylamine ligands," Journal of Colloid and Interface Science, vol. 460, pp. 164-172, 2015.

[48] B. W. Shiau, C. H. Lin, Y. Y. Liao et al., "The characteristics and mechanisms of Au nanoparticles processed by functional centrifugal procedures," Journal of Physics and Chemistry of Solids, vol. 116, pp. 161-167, 2018.

[49] H. Tsunoyama, N. Ichikuni, H. Sakurai, and T. Tsukuda, "Effect of electronic structures of Au clusters stabilized by poly( $N$-vinyl-2-pyrrolidone) on aerobic oxidation catalysis," Journal of the American Chemical Society, vol. 131, no. 20, pp. 7086-7093, 2009.

[50] M. Aflori, C. Miron, M. Dobromir, and M. Drobota, "Bactericidal effect on Foley catheters obtained by plasma and silver nitrate treatments," High Performance Polymers, vol. 27, no. 5, pp. 655-660, 2015.

[51] Y. F. Han, Z. Zhong, K. Ramesh, F. Chen, and L. Chen, "Effects of different types of $\gamma-\mathrm{Al}_{2} \mathrm{O}_{3}$ on the activity of gold nanoparticles for CO oxidation at low-temperatures," Journal of Physical Chemistry C, vol. 111, no. 7, pp. 3163-3170, 2007.

[52] J. Radnik, C. Mohr, and P. Claus, "On the origin of binding energy shifts of core levels of supported gold nanoparticles and dependence of pretreatment and material synthesis," Physical Chemistry Chemical Physics, vol. 5, no. 1, pp. 172177, 2003.

[53] M. A. Kostiainen, P. Hiekkataipale, A. Laiho et al., "Electrostatic assembly of binary nanoparticle superlattices using protein cages," Nature Nanotechnology, vol. 8, no. 1, pp. 52-56, 2013.

[54] M. Aflori, M. Butnaru, B. M. Tihauan, and F. Doroftei, "Ecofriendly method for tailoring biocompatible and antimicrobial surfaces of poly-l-lactic acid," Nanomaterials, vol. 9, no. 3, p. $428,2019$.

[55] M. Gosau, M. Haupt, S. Thude, M. Strowitzki, B. Schminke, and R. Buergers, "Antimicrobial effect and biocompatibility of novel metallic nanocrystalline implant coatings," Journal of Biomedical Materials Research Part B: Applied Biomaterials, vol. 104, no. 8, pp. 1571-1579, 2016.

[56] C. Xu, F. Yang, S. Wang, and S. Ramakrishna, "In vitro study of human vascular endothelial cell function on materials with various surface roughness," Journal of Biomedical Materials Research, vol. 71, no. 1, pp. 154-161, 2004.

[57] C. S. Ranucci and P. V. Moghe, "Substrate microtopography can enhance cell adhesive and migratory responsiveness to matrix ligand density," Journal of Biomedical Materials Research, vol. 54, no. 2, pp. 149-161, 2001. 
[58] J. L. Maitre and C. P. Heisenberg, "The role of adhesion energy in controlling cell-cell contacts," Current Opinion in Cell Biology, vol. 23, no. 5, pp. 508-514, 2011.

[59] S. Giljean, M. Bigerelle, and K. Anselme, "Roughness statistical influence on cell adhesion using profilometry and multiscale analysis," Scanning, vol. 36, no. 1, pp. 2-10, 2014.

[60] R. G. Richards, "The effect of surface roughness on fibroblast adhesion in vitro," Injury, vol. 27, Supplement 3, pp. S/C38S/C43, 1996.

[61] R. V. Goreham, A. Mierczynska, L. E. Smith, R. Sedev, and K. Vasilev, "Small surface nanotopography encourages fibroblast and osteoblast cell adhesion," RSC Advances, vol. 3, no. 26, pp. 10309-10317, 2013.

[62] L. Chen, J. Sun, Z. Zhu et al., "The adhesion and proliferation of bone marrow-derived mesenchymal stem cells promoted by nanoparticle surface," Journal of Biomaterials Applications, vol. 27, no. 5, pp. 525-536, 2013.

[63] M. Staszek, J. Siegel, S. Rimpelová, O. Lyutakov, and V. Švorčík, "Cytotoxicity of noble metal nanoparticles sputtered into glycerol," Materials Letters, vol. 158, pp. 351-354, 2015. 